\section{Utility of Echocardiography for the Diagnosis of Right Atrial Dissection}

Xiaojing Ma, MD; Zhenxing Sun, MD; Juan Xia, MD; Xiaomi Guo, MD; Mingxing Xie, MD, PhD


17-year-old girl presented with chief complaints of intermittent palpitations and chest tightness for 2 years. Transthoracic echocardiography revealed a large aneurysmal sac in the right atrium surrounded by a membrane-like structure, which compressed the tricuspid valve (Figure A,B; Supplementary Movie 1). Contrast echocardiography detected communication of the aneurysmal sac with the atrial true lumen (Figure C; Supplementary Movie 2). We diagnosed the membrane-like structure as right atrial (RA) dissection. Further inquiry with contrastenhanced computed tomography (Figure D-F) and cardiac magnetic resonance imaging (Figure G) confirmed the diagnoses of RA dissection. The pre-bypass transesophageal echocardiography showed the size and location of the RA dissection (Figure H,I). Because of a concern of potential arrhythmias and thromboembolic complications, we recommended operative resection of the RA dissection. The aneurysmal sac was completely resected (Figure $\mathbf{J}, \mathbf{K}$ ), and the lateral wall of RA was reconstructed. Histological examination of the resected RA tissue was consistent with the diagnosis of RA dissection (Figure $\mathbf{L}$ ).

To the best of our knowledge, left atrial dissection has been reported in a small number of patients, but cases of RA dissection are rare. This case highlights that echocardiography plays a key role in the accurate characterization of RA dissection.

\section{Acknowledgments}

We acknowledge the National Natural Science Foundation of China

Received January 6, 2022; revised manuscript received January 19, 2022; accepted January 22, 2022; J-STAGE Advance Publication released online February 16, 2022 Time for primary review: 4 days

Department of Ultrasound, Wuhan Asia Heart Hospital, Wuhan (X.M., J.X., X.G.); Department of Ultrasound, Wuhan Asia General Hospital, Wuhan (X.M., X.G.); and Department of Ultrasound Medicine, Union Hospital, Tongji Medical College, Huazhong University of Science and Technology, Wuhan (Z.S., M.X.), China

The first four authors contributed equally to this work (X.M., Z.S., J.X., X.G.).

Mailing address: Mingxing Xie, MD, PhD, Department of Ultrasound Medicine, Union Hospital, Tongji Medical College, Huazhong University of Science and Technology, Wuhan, 430022, China. E-mail: xiemx@hust.edu.cn

All rights are reserved to the Japanese Circulation Society. For permissions, please e-mail: cj@j-circ.or.jp ISSN-1346-9843
(No. 81701716; 81727805), Scientific Research Program of Wuhan Municipal Health Commission (WX21D58; WX21Q30), and the Wuhan Science and Technology Bureau (2019020701011422).

\section{Supplementary Files}

Supplementary Movie 1. Transthoracic echocardiography demonstrating right atrial dissection in the presence of giant right aneurysmal sac.

Supplementary Movie 2. Contrast Echocardiography showing microbubbles filling simultaneously the giant right aneurysmal sac and the atrial true lumen.

Please find supplementary file(s);

http://dx.doi.org/10.1253/circj.CJ-21-1070

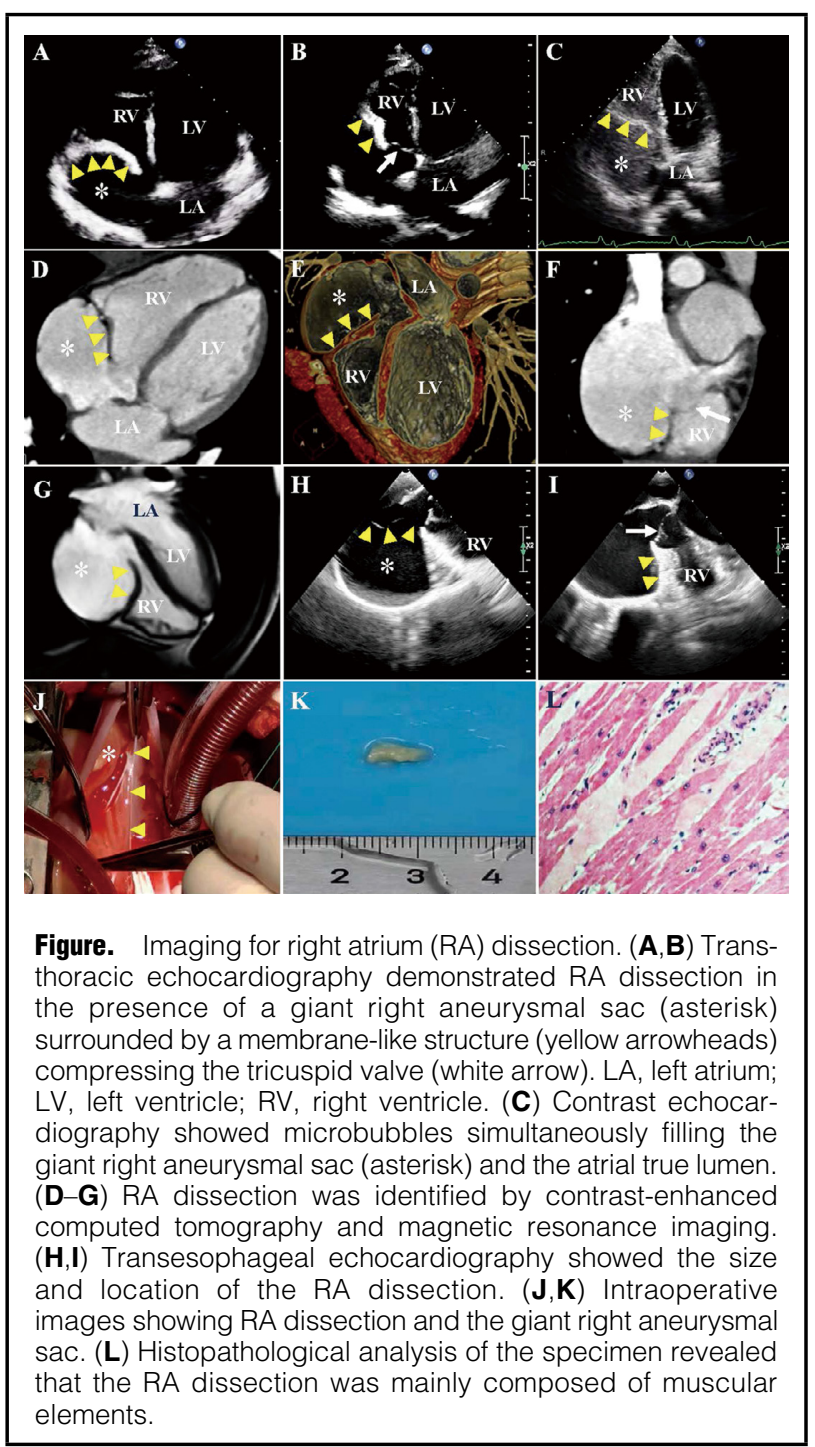

